\title{
ON THE STRESS FREE DEFORMATION OF LINEAR FGM INTERFACE UNDER CONSTANT TEMPERATURE
}

\author{
Artur GANCZARSKI*, Damian SZUBARTOWSKI* \\ *Institute of Applied Mechanics, Department of Mechanical Engineering, Cracow University of Technology, \\ 31-864 Kraków, al. Jana Pawła II 37, Poland
}

artur.ganczarski@pk.edu.pl, tszubartowski@o2.pl

received 24 February 2015, revised 9 October 2015, accepted 14 October 2015

\begin{abstract}
This paper demonstrates the stress free thermo-elastic problem of the FGM thick plate. Existence of such a purely thermal deformation is proved in two ways. First proof is based on application of the lljushin thermo-elastic potential to displacement type system of equations. This reduces 3D problem to the plane stress state problem. Next it is shown that the unique solution fulfils conditions of simultaneous constant temperature and linear gradation of thermal expansion coefficient. Second proof is based directly on stress type system of equations which straightforwardly reduces to compatibility equations for purely thermal deformation. This occurs if only stress field is homogeneous in domain and at boundary. Finally an example of application to an engineering problem is presented.
\end{abstract}

Key words: Linear FGM Interface, Stress Free Deformation

\section{INTRODUCTION}

Functionally graded materials (FGMs) provide thermal insulation and mechanical toughness at high temperature by varying the composition of thermal conductivity coefficient, thermal expansion coefficient and Young's modulus from high temperature side to low temperature side continuously and simultaneously by removing the discontinuity of layered plate. These advantages cause that FGMs are applicable in many fields such as high performance engines for aerospace vehicles, turbine blades and heat-resisting tools. A general overview of thermal stresses in FGMs comprises work by Noda (1999).

Numerous analytical solutions of thermo-elastic plane or three-dimensional problems of FGMs take advantage of specific power or exponential function approximation methods of multilayered composite plate, limiting simultaneously their generality and suggesting question how to reduce the problem. One way to attain this may be proving theorem on the stress free deformation accompanying linear gradation of thermo-mechanical properties of the material staying in constant temperature condition. Such a proof can be done following two ways taking advantage of either displacement or stress formulation of thermo-elastic equations. In the first case lemma consists in generalization of theorem on the plane stress state in an isotropic thermo-elastic thick plate, originally proved by Sneddon and Lockett (1960). The authors presented convinced proof for a problem of semi-infinite thermoelastic medium bounded by two parallel planes and loaded by an arbitrary temperature field on one surface. The method of solution employed was the double Fourier transform. The results confirmed solution of analogous problems, being inspiration to their work, received earlier by Sternberg and McDowell (1957), based on Green's function, and by Muki (1957), who used method combining the theory of Fourier series and the Hankel transforms of integral order. Also there exists other more elegant way, based on application of lljushin's potential (lljushin et al., 1979), which is demonstrated in the present work. Final step of the proof of theorem relays on pointing out that unique solution of the plane stress equations, that satisfy homogeneous boundary conditions, guarantees stress free deformation if only temperature field is constant and gradation of thermal expansion coefficient is linear function.

In the other case, when stress formulation of thermo-elastic equations is used, the proof of theorem is almost elementary and turns out to be straightforward analogy to these which were done by Fung (1965) and Nowacki (1970) for homogeneous material.

\section{FGM'S - CONCEPT, FABRICATION, PROPERTIES AND NUMERICAL MODELING}

In many applications, especially in the space industry as well as electronic industry, structures or part of structures are exposed to high temperature, usually up to $2000 \mathrm{~K}$ or even $3500 \mathrm{~K}$ in some parts of rocket engines, see Schulz et al. (2003), high temperature gradients, and/or cyclic temperature changes. Conventional metallic materials, such as carbon steels or stainless steels: ASTM 321, ASTM 310, nickel- or aluminium-based alloys cannot resist such high temperatures, see Odqvist (1966). The first method to improve the resistance of metallic structures against extreme temperature conditions consists in covering the structure with a ceramic layer since ceramics are known for their high thermal resistance. For instance, in a metal-ceramic composite: Al-SiC the thermal conductivities ratio is approximately equal: $\lambda_{\mathrm{m}} / \lambda_{\mathrm{c}}=3.6$, the thermal expansion coefficients ratio: $\alpha_{\mathrm{m}} / \alpha_{\mathrm{c}}=5$, whereas the elastic moduli ratio: $E_{m} / E_{\mathrm{c}}=0.16$, see Potarescu and Sugano (1993). Indices $m$ and $c$ refer to matrix and ceramic mate- 
rials respectively. Hence, at the metal-ceramic interface, severe discontinuity of thermo-mechanical properties occurs, which results in high strain and stress mismatch at the interface. As a consequence, delamination or failure of the coating is rapidly observed. As a remedy to these disadvantages the concept of Functionally Graded Materials - FGM, was developed in Japan in the 1980s, see Yamanouchi et al. (1990), giving structural components a spatial gradient in thermo-mechanical properties. The spatial gradient is achieved by use of two-component composites. The volume fraction of the composite constituents varies spatially such that the effective thermo-mechanical properties change smoothly from one material (ceramic) to the other (metal). In this way, in the case of a Thermal Barrier Coating deposited on a metallic substrate, the heat-resistant ceramic layer and the solid metal are separated by functionally graded FG layer, the composition of which varies from pure ceramic to pure metal. The processing technologies for TBCs and FGMs may lead to residual stresses, which are built-in during cool-down from the elevated fabrication temperature. These residual stresses may be significant relative to thermo-mechanical stresses applied subsequently. As regards FG layer processing, Plasma Spray Thermal Barrier Coating leads to lamellar microstructures, whereas columnarlamellar microstructures are produced when using Electron Beam Physical Vapour Deposition, see Lee et al. (1996), Schulz et al. (2003).

A general review article on the application of the several ceramic materials to TBCs is given by Lee et al. (1996). Selected thermo-mechanical properties as elastic modulus $E$ and both thermal expansion $\alpha$ and conductivity $\lambda$ coefficients are summarized in Table 1 for two alumina-based composites, see Chen and Tong (2004), Cho and Shin (2004) for $\mathrm{Ni}_{-} \mathrm{Al}_{2} \mathrm{O}_{3}$ and Wang et al. (2000) for $\mathrm{Ti}_{-}-\mathrm{Al}_{2} \mathrm{O}_{3}$.

Tab. 1. Comparison of properties of constituents of two alumina-based

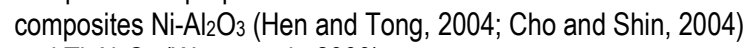

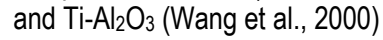

\begin{tabular}{|c|c|c|c|}
\hline Composite & $E[\mathrm{GPa}]$ & $\lambda[\mathrm{W} / \mathrm{mK}]$ & $\alpha \cdot 10^{-6}[1 / \mathrm{K}]$ \\
\hline $\mathrm{Ni}$ & 199.5 & 90.7 & 13.3 \\
$\mathrm{Al}_{2} \mathrm{O}_{3}$ & 393.0 & 30.7 & 8.8 \\
\hline $\mathrm{Al}$ & 73 & 154 & 23 \\
$\mathrm{Al}_{2} \mathrm{O}_{3}$ & 380 & 46 & 8.5 \\
\hline
\end{tabular}

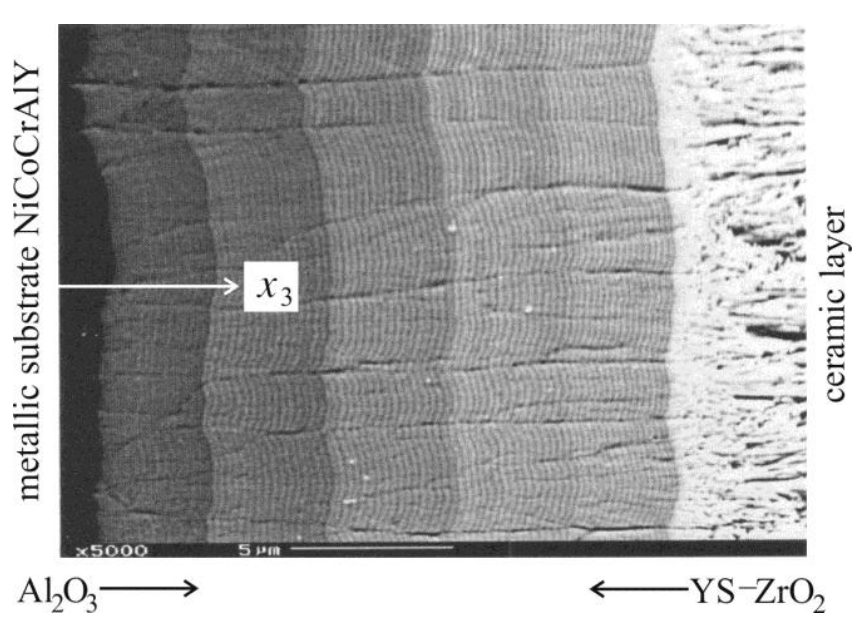

Fig. 1. Microstructure of chemically graded Electron Beam Physical Vapour Deposition thermal barrier coating, after Schulz et al. (2003)
When the classical FEM based on homogeneous elements is used for FGMs, the material properties stay the same for all integration points belonging to one finite element. This means that material properties may vary in a piecewise continuous manner, from one element to the other and a unique possibility to model FGM structure is approximation by use of appropriately fine mesh. On the other hand, a too coarse mesh may lead to unrealistic stresses at the interface between the subsequent layers. To overcome this difficulty a special graded element has been introduced by Kim and Paulino (2002) to discretize FGM properties. The material properties at Gauss quadrature points are interpolated there from the nodal material properties by the use of isoparametric interpolation functions. Contrary to the classical FEM formulation, the stiffness matrix of an element is expressed by the integral in which constitutive matrix is a function of the coordinates. In the original formulation the same shape functions are used for approximation of the displacement field and material inhomogeneity. However, from the numerical point of view nothing stands in the way of implementation of shape functions referring directly to the individual character of inhomogeneity, for instance power functions, see Akai et al. (2005) or exponential functions, see Bagri et al. (2005).

\section{THE GENERAL FORMULATION OF FGM THERMO-ELASTIC PROBLEM}

A thermo-elastic body under consideration (Fig.1) is bounded by two parallel planes normal to axis $x_{3}$, and its thermomechanical properties such as thermal conductivity coefficient, thermal expansion coefficient and Young's (Kirchhoff's) modulus are optional functions of $x_{3}$

$\lambda=\lambda\left(x_{3}\right) \quad \alpha=\alpha\left(x_{3}\right) \quad E=E\left(x_{3}\right) \quad G=G\left(x_{3}\right)$.

Since $E\left(x_{3}\right)$ and $G\left(x_{3}\right)$ are controlled by the same function of $x_{3}$ the Poisson ratio is considered as independent of $x_{3}$ and satisfying classical relation

$v=\frac{E}{2 G}-1$.

This guarantees isotropy (two independent material constants) on one hand and simultaneously prevents from some peculiar effects occurring on the other hand, see Ganczarski and Barwacz (2004).

The body is established a temperature field $T+\theta\left(x_{i}\right)$, where $T$ stands for the temperature of the solid corresponding to zero stress and strain. Also it is assumed that there are no body forces within the solid and that its surfaces are free from tractions.

The system of equations of uncoupled thermo-elasticity expressed in displacements takes the form

$$
\begin{aligned}
& \nabla^{2} u_{i}+\frac{1}{1-2 v} \frac{\partial \Theta}{\partial x_{i}}+\frac{1}{\underline{G} \frac{\partial G}{\partial x_{3}}\left(\frac{\partial u_{i}}{\partial x_{3}}+\frac{\partial u_{3}}{\partial x_{i}}\right)}=2 \frac{1+v}{1-2 v} \frac{\partial(\propto \theta)}{\partial x_{i}}, \\
& \nabla^{2} u_{3}+\frac{1}{1-2 v} \frac{\partial \Theta}{\partial x_{3}}+\frac{2}{G} \frac{\partial G}{\partial x_{3}}\left(\frac{\partial u_{3}}{\partial x_{3}}+\frac{v \Theta}{1-2 v}\right)=2 \frac{1+v}{1-2 v} \\
& \times\left[\frac{\partial(\alpha \theta)}{\partial x_{3}}+\frac{1}{E} \frac{\partial E}{\partial x_{3}} \alpha \theta\right], \\
& \nabla^{2} \theta+\frac{1}{\lambda} \frac{\partial \lambda}{\partial x_{3}} \frac{\partial \theta}{\partial x_{3}}=0,
\end{aligned}
$$

where $u_{i}$ denotes the displacement vector, and $\Theta=\operatorname{grad}\left(u_{i}\right)$ 
is the dilatation. The underlined terms in Eqs (3) yield of FGM application and they are additional in comparison with classical formulation of homogeneous material. The relation between the stress tensor $\sigma_{i j}$ and the displacement vector $u_{i}$ is given by the Duhamel-Neumann equation

$\sigma_{i j}=G\left[\frac{\partial u_{i}}{\partial x_{j}}+\frac{\partial u_{j}}{\partial x_{i}}+2\left(\frac{v(\Theta-3 \alpha \theta)}{1-2 v}-\alpha \theta\right) \delta_{i j}\right]$.

The variation of temperature $\theta$ throughout the solid is determined by steady Fourier equation Eq. $\left(3_{3}\right)$ in case of absence of inner heat sources.

System of equations expressed in stresses (extension of Beltrami-Michell formulation) equivalent to (3) is as follows

$$
\begin{gathered}
\nabla^{2} \sigma_{i i}+\frac{1}{1+v}\left[\frac{\partial^{2} s}{\partial x_{i}^{2}}-\nabla^{2} s\right]-2 E \frac{\partial}{\partial x_{3}}\left(\frac{1}{E}\right)\left[\frac{\partial \sigma_{j j}}{\partial x_{3}}-\frac{v}{1+v} \frac{\partial s}{\partial x_{3}}\right] \\
-E \frac{\partial^{2}}{\partial x_{3}^{2}}\left(\frac{1}{E}\right)\left[\sigma_{j j}-\frac{v}{1+v} s\right] \\
+\frac{E}{1+v}\left[\frac{\partial^{2}(\alpha \theta)}{\partial x_{i}^{2}}-\nabla^{2}(\alpha \theta)\right]=0 \quad i, j=1,2,
\end{gathered}
$$

$\nabla^{2} \sigma_{33}+\frac{1}{1+v}\left[\frac{\partial^{2} s}{\partial x_{3}^{2}}-\nabla^{2} s\right]+\frac{E}{1+v}\left[\frac{\partial^{2}(\alpha \theta)}{\partial x_{3}^{2}}-\nabla^{2}(\alpha \theta)\right]=0$,

$$
\begin{gathered}
\nabla^{2} \tau_{i j}+\frac{1}{1+v} \frac{\partial^{2} s}{\partial x_{i} x_{j}}+\frac{\partial}{\partial x_{3}}\left(\frac{1}{E}\right)\left[\frac{\partial \sigma_{k k}}{\partial x_{i}}-\frac{1}{1+v} \frac{\partial s}{\partial x_{i}}-\frac{\partial \tau_{k i}}{\partial x_{k}}\right] \\
+\frac{E}{1+v} \frac{\partial^{2}(\alpha \theta)}{\partial x_{i} x_{j}}=0 \quad i, j, k=1,2,3 \quad i \neq j \neq k
\end{gathered}
$$

$\nabla^{2} \theta+\frac{1}{\lambda} \frac{\partial \lambda}{\partial x_{3}} \frac{\partial \theta}{\partial x_{3}}=0$,

where $s=\operatorname{tr}(\sigma)=\sigma_{11}+\sigma_{22}+\sigma_{33}$. It worth to notice that equations (51-3) can be obtained either in classical way or directly from equations (31-2) according to concept by lgnaczak (1959).

\section{CONDITIONS OF EXISTENCE OF STRESS FREE DEFORMATION}

\subsection{Proof based on displacement formulation}

To solve Eqs (3) the following potential, originally proposed by lljushin et al. [8], is introduced

$$
\begin{aligned}
& u_{i}=\frac{\partial \phi}{\partial x_{i}}, \quad u_{3}=-\frac{\partial \phi}{\partial x_{3}}+f\left(x_{3}\right) \\
& f\left(x_{3}\right)=A x_{3}^{2}+B x_{3}+C \\
& \alpha \theta=2 \frac{1-v}{1+v} A x_{3}-\frac{1}{1+v} \frac{\partial^{2} \phi}{\partial x_{3}^{2}},
\end{aligned}
$$

where function of displacement potential $\phi$ is of harmonic type

$\nabla^{2} \phi=0$

and $A, B$, and $C$ are constants.

Simple introducing of definitions (6) to Eqs (3) shows that only equations of mechanical state are satisfied as identity, contrary to the case of homogeneous material, when also the equation of thermal state is satisfied as identity

$$
\begin{aligned}
& \frac{\partial}{\partial x_{i}}(\underbrace{\left.\nabla^{2} \phi\right)}_{=0}+\frac{1}{1-2 v} \frac{\partial}{\partial x_{i}}(\underbrace{\nabla^{2} \phi}_{=0}-2 \frac{\partial^{2} \phi}{\partial x_{3}^{2}}+2 A x_{3}+B) \\
& +\frac{1}{G} \frac{\partial G}{\partial x_{3}} \underbrace{\left(\frac{\partial^{2} \phi}{\partial x_{3} \partial x_{i}}-\frac{\partial^{2} \phi}{\partial x_{i} \partial x_{3}}\right)}_{=0} \\
& =2 \frac{1+v}{1-2 v}\left(-\frac{1}{1+v} \frac{\partial^{3} \phi}{\partial x_{i} \partial x_{3}^{2}}\right)-\frac{\partial}{\partial x_{3}}(\underbrace{\left.\nabla^{2} \phi\right)}_{=0}+2 A \\
& +\frac{1}{1-2 v} \frac{\partial}{\partial x_{3}}(\underbrace{\nabla^{2} \phi}_{=0}-2 \frac{\partial^{2} \phi}{\partial x_{3}^{2}}+2 A x_{3}+B) \\
& +\frac{2}{G} \frac{\partial G}{\partial x_{3}}\left[-\frac{\partial^{2} \phi}{\partial x_{3}^{2}}+2 A x_{3}+B+\frac{v}{1-2 v}(\underbrace{\nabla^{2} \phi}_{=0}-2 \frac{\partial^{2} \phi}{\partial x_{3}^{2}}\right. \\
& \left.\left.+2 A x_{3}+B\right)\right]=2 \frac{1+v}{1-2 v}\left[2 \frac{1-v}{1+v} A-\frac{1}{1+v} \frac{\partial^{3} \phi}{\partial x_{3}^{3}}\right. \\
& \left.+\frac{1}{E} \frac{\partial E}{\partial x_{3}}\left(2 \frac{1-v}{1+v} A x_{3}-\frac{1}{1+v} \frac{\partial^{2} \phi}{\partial x_{3}^{2}}\right)\right] .
\end{aligned}
$$

The stress components referring to the plane stress state with respect to axis $x_{3}$

$$
\begin{aligned}
\tau_{13} & =G\left(\frac{\partial u_{1}}{\partial x_{3}}+\frac{\partial u_{3}}{\partial x_{1}}\right)=G \underbrace{\left(\frac{\partial^{2} \phi}{\partial x_{1} \partial x_{3}}-\frac{\partial^{2} \phi}{\partial x_{3} \partial x_{1}}\right)}_{=0}=0, \\
\tau_{23} & =G\left(\frac{\partial u_{2}}{\partial x_{3}}+\frac{\partial u_{3}}{\partial x_{2}}\right)=G \underbrace{\left(\frac{\partial^{2} \phi}{\partial x_{2} \partial x_{3}}-\frac{\partial^{2} \phi}{\partial x_{3} \partial x_{2}}\right)}_{=0}=0, \\
\sigma_{33} & =2 G\left[\frac{\partial u_{3}}{\partial x_{3}}-\alpha \theta+\frac{v}{1-2 v}(\Theta-3 \alpha \theta)\right] \\
& =2 G\left\{-\frac{\partial^{2} \phi}{\partial x_{3}^{2}}+2 A x_{3}+B-2 \frac{1-v}{1+v} A x_{3}+\frac{1}{1+v} \frac{\partial^{2} \phi}{\partial x_{3}^{2}}\right. \\
& +\frac{v}{1-2 v}[\underbrace{\nabla^{2} \phi}_{=0}-2 \frac{\partial^{2} \phi}{\partial x_{3}^{2}}+2 A x_{3}+B \\
& \left.\left.-3\left(2 \frac{1-v}{1+v} A x_{3}-\frac{1}{1+v} \frac{\partial^{2} \phi}{\partial x_{3}^{2}}\right)\right]\right\}=2 G \frac{1-v}{1-2 v} B,
\end{aligned}
$$

are also identically equal to zero when $B=0$ for any point $x_{i}$. This proves that Eqs (6) transform original mechanical problem Eq. (3) into plane stress problem

$\nabla^{2} \phi+2(1-v) A x_{3}-(1+v) \alpha \theta=0$.

The general solution (10) can be written in a form which is more suitable to plate problem, namely in which the thermoelastic solid is bounded by two parallel planes $x_{3}=z$ and exhibits axial symmetry

$\frac{\partial^{2} \phi}{\partial r^{2}}+\frac{1}{r} \frac{\partial \phi}{\partial r}+2(1-v) A z-(1+v) \alpha \theta=0$.

Differentiation of Eq. (11) with respect to $r$ and next substitution $u=\partial \phi / \partial r$ according to Eqs (61), lead to the classical Euler-type differential equation describing thermo-mechanical membrane state

$\frac{\partial^{2} u}{\partial r^{2}}+\frac{1}{r} \frac{\partial u}{\partial r}-\frac{u}{r^{2}}=(1+v) \frac{\partial(\alpha \theta)}{\partial r}$.

Unique solution of equation (12) that satisfies homogeneous boundary conditions

$u(0)=0, \quad \sigma_{r}(R)=0$

takes well known form

$u=\frac{(1-v)}{R^{2}} r \int_{0}^{R} \alpha \theta r \mathrm{~d} r+\frac{(1+v)}{r} \int_{0}^{r} \alpha \theta \rho \mathrm{d} \rho$, 
$\sigma_{r}=\frac{E}{R^{2}} \int_{0}^{R} \alpha \theta r \mathrm{~d} r-\frac{E}{r^{2}} \int_{0}^{r} \alpha \theta \rho \mathrm{d} \rho$,

$\sigma_{\varphi}=\frac{E}{R^{2}} \int_{0}^{R} \alpha \theta r \mathrm{~d} r+\frac{E}{r^{2}} \int_{0}^{r} \alpha \theta \rho \mathrm{d} \rho-E \alpha \theta$,

which in case of constant temperature $\theta=$ const and linear gradation of coefficient of thermal expansion $\alpha(z)=a_{0}+a_{1} z$ leads to purely linear (stress-less) deformation

$u(r, z)=\alpha \theta(z) r, \quad \sigma_{r}=\sigma_{\varphi} \equiv 0$,

what closes the proof.

\subsection{Proof based on stress formulation}

The proof of theorem presented in point 4.1, in case of the stress formulation Eq. (51-3), is straightforward analogy to those done by Fung (1965) and Nowacki (1970) for homogenous material. This turns out to be almost elementary when one assumes that $\sigma_{i j} \equiv 0$ in both Eq. (51-3) and appropriate boundary conditions. Namely, system of equations is satisfied as identity if

$$
\begin{array}{ll}
\frac{\partial^{2}(\alpha \theta)}{\partial x_{i}^{2}}-\nabla^{2}(\alpha \theta)=0 & i=1,2,3, \\
\frac{\partial^{2}(\alpha \theta)}{\partial x_{i} x_{j}}=0 & i, j=1,2,3,
\end{array}
$$

$\nabla^{2} \theta+\frac{1}{\lambda} \frac{\partial \lambda}{\partial x_{3}} \frac{\partial \theta}{\partial x_{3}}=0$

For constant temperature $\theta=$ const satisfying Fourier's law $\left(16_{3}\right)$ the unique solution of $\left(16_{1,2}\right)$ corresponds again to the linear gradation of coefficient of thermal expansion $\alpha\left(x_{3}\right)=a_{0}+$ $a_{1} x_{3}$.

\section{EXAMPLE}

It has been proved in points 4.1 and 4.2 that material of linear gradation of thermal expansion coefficient, subjected to constant temperature exclusively, is not stressed. This means that it exhibits unconstrained and purely thermal deformation. In case of axial symmetry such deformation can be expressed by following equations

$\varepsilon_{r}=\frac{\partial u}{\partial r}=\alpha \theta, \quad \varepsilon_{\varphi}=\frac{u}{r}=\alpha \theta, \quad \varepsilon_{z}=\frac{\partial w}{\partial z}=\alpha \theta$.

Let us assume that the structure is composed of homogeneous metallic substrate (Al) and ceramic layer $\left(\mathrm{Al}_{2} \mathrm{O}_{3}\right)$, joined by FGM interface as shown in Fig.3, and thermo-elastic properties presented in Tab.1.

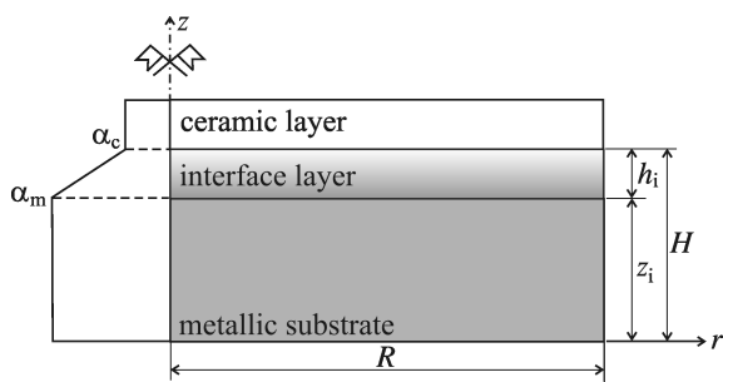

Fig. 3. Metallic substrate and ceramic layer joined by FGM interface of linear thermal expansion coefficient structure
Hence linearly graded coefficient of thermal expansion exhibits polygonal function

$\alpha(z)= \begin{cases}\alpha_{\mathrm{m}} & 0 \leq z<z_{\mathrm{i}} \\ \alpha_{\mathrm{m}}-\left(\alpha_{\mathrm{m}}-\alpha_{\mathrm{c}}\right) \frac{z-z_{\mathrm{i}}}{h_{\mathrm{i}}} & z_{\mathrm{i}} \leq z<z_{\mathrm{i}}+h_{\mathrm{i}} \\ \alpha_{\mathrm{c}} & z_{\mathrm{i}}+h_{\mathrm{i}} \leq z \leq H\end{cases}$

and we easily arrive at following of solution Eqs (18) for $u$

$u(r, z)=\int_{0}^{r} \alpha(z) \theta \mathrm{d} \rho=$
$\begin{cases}\alpha_{\mathrm{m}} \theta r & 0 \leq z<z_{\mathrm{i}} \\ \alpha_{\mathrm{m}} \theta r-\left(\alpha_{\mathrm{m}}-\alpha_{\mathrm{c}}\right) \frac{z-z_{\mathrm{i}}}{h_{\mathrm{i}}} \theta r & z_{\mathrm{i}} \leq z<z_{\mathrm{i}}+h_{\mathrm{i}} \\ \alpha_{\mathrm{c}} \theta r & z_{\mathrm{i}}+h_{\mathrm{i}} \leq z \leq H\end{cases}$

and for $w$ respectively

$w(r, z)=\int_{0}^{z} \alpha(z) \theta \mathrm{d} \zeta=$
$\left\{\begin{array}{cl}\alpha_{\mathrm{m}} \theta z & 0 \leq z<z_{\mathrm{i}} \\ \alpha_{\mathrm{m}} \theta z-\left(\alpha_{\mathrm{m}}-\alpha_{\mathrm{c}}\right) \frac{\left(z-z_{\mathrm{i}}\right)^{2}}{2 h_{\mathrm{i}}} \theta & z_{\mathrm{i}} \leq z<z_{\mathrm{i}}+h_{\mathrm{i}} \\ \alpha_{\mathrm{c}} \theta\left(z_{\mathrm{i}}+h_{\mathrm{i}}\right)-\left(\alpha_{\mathrm{m}}-\alpha_{\mathrm{c}}\right) \frac{h_{\mathrm{i}}}{2} \theta & z_{\mathrm{i}}+h_{\mathrm{i}} \leq z \leq H \\ +\alpha_{\mathrm{c}} \theta\left(z-z_{\mathrm{i}}+h_{\mathrm{i}}\right) & \end{array}\right.$.

The displacement field corresponding to stress free deformation defined by Eqs (19-20) is spanned over the mesh of $81 \times 41$ square elements and shown in Fig. 4. It is well visible that both substrate and ceramic layers exhibit homogeneous deformation, whereas deformation of interface links them satisfying simultaneously stress less state.

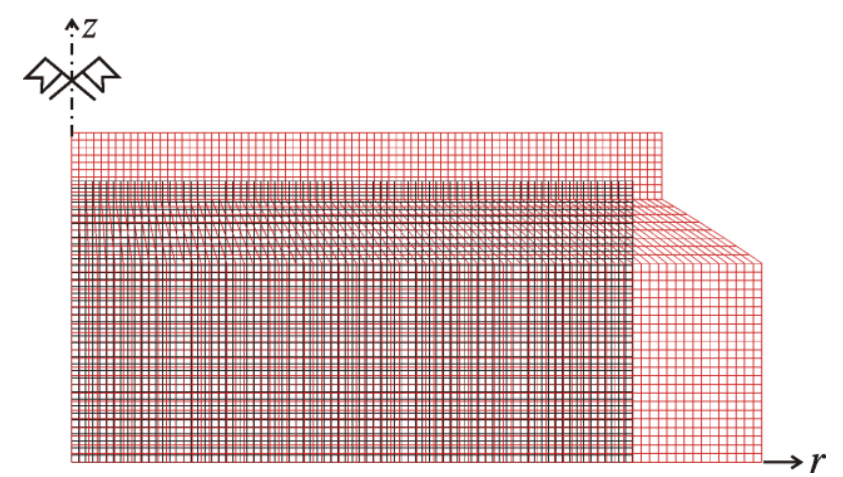

Fig. 4. Unconstrained (stress less) and purely thermal deformation Eqs (19-20) of three layer structure: initial mesh - black colour, deformed mesh - red colour (displacement magnified $\times 100$ )

\section{CONCLUSIONS}

Homogeneous temperature field does not result stress in thermo-elastic material of linear gradation, if only force type boundary conditions are homogeneous and there are not body forces. However, the case of stress less deformation has only theoretical sense since neither manufacturing nor classical FEM do not allow for modeling of continuously varying FGM. Namely, from technological point of view the $\mathrm{Al}_{2} \mathrm{O}_{3}$ outer layer deposed on top of a NiCoCAlY bond coat, shown in Fig. 1 after Schulz et al. (2003), exhibits hardly noticeable stress state resulting from mismatch between metal and ceramic Young's modules and coefficients of thermal expansion. On the other hand, if the classical FEM is used for solving FGM problems, the material properties can only vary in a piecewise continuous manner since all integra- 
tion points within an element have a common property value. To overcome this difficulty a special graded element concept, based on additional interpolation for nodal material properties, is necessary to apply.

\section{REFERENCES}

1. Akai T., Ootao Y., Tanigawa Y. (2005), Piezothermoelastic analysis of functionally graded piezoelectric cylindrical panel due to nonuniform heat supply in the circumefernetial direction, Proc. Thermal Stresses'05, 709-712.

2. Bagri A., Eslami M. R., Samsam-Shariat B. A. (2005), Coupled thermoelasticity of functionally graded layer, Proc. Thermal Stresses'05, 721-724.

3. Chen W. F., Tong L. (2004), Sensitivity analysis of heat conduction for functionally graded materials, Mater. Design, 25, 633-672.

4. Cho J. R., Shin S. W. (2004), Material composition optimization for heat-resisting FGMs by artificial neural network, Composites, A35, 585-595.

5. Fung Y. C. (1965), Foundations of solid mechanics, Prentice-Hall, New Jersey.

6. Ganczarski A., Barwacz L. (2004), Notes on damage effect tensors of two-scalar variables, Int. J. Damage Mech., 13, 3, 287-295.

7. Ignaczak J. (1959), Direct determination of stresses from the stress equations of motion in elasticity, Arch. Mech. Stos., 11(5), 671-678.

8. Iljushin A. A., Lomakin W. A., Shmakov A. P. (1979), Mechanics of Continuous Media, Moscow.

9. Kim J. H., Paulino G. H. (2002), Isoparametric graded finite elements for non-homogeneous isotropic and orthotropic materials, ASME J. Appl. Mech., 69, 502-514.

10. Lee W. Y., Stinton D. P., Berndt C. C, Erdogan F., Lee Y.-D., Mutasin Z. (1996), Concept of functionally graded materials for advanced thermal barrier coating applications, J. Am. Ceram. Soc. 79, 3003-3012.
11. Muki R. (1957), Thermal stresses in a semi-infinite solid and a thick plate under steady distribution of temperature, Proc. Fac. Eng. Keio. Univ., 9, 42.

12. Noda N. (1999), Thermal stresses in functionally graded materials, J. Thermal Stresses, Vol. 22(4/5), 477-512.

13. Nowacki W. (1970), Theory of elasticity, PWN, Warsaw.

14. Odqvist F. K. G. (1966), Mathematical theory of creep and creep rupture, Oxford, Clarendon Press.

15. Potarescu F., Sugano Y. (1993), An improved solution to thermoelastic material design in fuctionally graded materials: Scheme to reduce thermal stresses, Comput. Mech. Appl. Mech.Eng., 109, 377-389.

16. Schulz U., Bach F. W., Tegeder G. (2003), Graded coating for thermal, wear and corrosion barriers, Mater. Sci. Eng., A 362(1-2), 61-80.

17. Sneddon I. N., Lockett F. J. (1960), On the steady-state thermoelastic problem for the half-space and the thick plate, Quart. Appl. Math., Vol. 18(2), 145-153.

18. Sternberg E., McDowell E. L. (1957), On the steady-state thermoelastic problem for the half-space, Quart. Appl. Math., 14, 381.

19. Wang B.-L., Han J. C., Du S. Y. (2000), Crack problems for functionally graded materials under transient thermal loading, $\mathrm{J}$. Thermal Stresses, 23, 143-168.

20. Yamanouchi M., Hirai T., Shiota I. (1990), Overall view of the P/M fabrication of fuctionally gradient materials, Proc. First Int. Symp. Functionally Gradient Materials, eds Yamanouchi et al., Sendai, Japan, 59-64.

This work was supported by National Science Centre Poland grant $\mathrm{Nr}$ UMO-2011/03/B/ST8/05132. 